\title{
La tentation de la pensée raciale dans les lectures historio- graphiques françaises et allemandes 1920-1930 du peuplement dit germanique de la Gaule. Une conception historique de la race
}

L'analyse de la question du peuplement dit germanique au très haut Moyen Âge a très tôt confronté les historiens français et allemands à la notion de race, définie historiquement: Francs, Gaulois, et plus largement Germains peuvent-ils être appréhendés comme une ou plusieurs races, à quel titre, et quelles en seraient les conséquences historiques, notamment au regard de l'unité nationale? Le terme s'emploie dans la langue classique pour désigner une origine, notamment familiale, et spécifiquement quand elle est illustre (la race franque/mérovingienne). En 1727, le comte de Boulainvilliers systématise une théorie alors en latence dans les cercles érudits. La population de la France se diviserait en deux souches, nommées races, la galloromaine, asservie, et la franque, conquérante. La seconde, dont est issue la noblesse de l'époque, tire de son ancienne victoire sur la première son statut privilégié. Cette posture aristocratique ne remporte pas l'unanimité dans les milieux savants, elle apparaît subversive et réactionnaire et trouve chez Montesquieu, par exemple, une exposition plus nuancée. Le tournant démocratique de la fin du XVIII ${ }^{\mathrm{e}}$ siècle étend la souveraineté de la nation de la seule famille royale - et de l'aristocratie - à l'ensemble de la population. Pourtant, la thèse des deux races trouve encore des partisans au début du $\mathrm{XIX}^{\mathrm{e}}$ siècle, et Augustin Thierry en inverse la logique: la conquête franque puis la royauté mérovingienne ont en fait usurpé le pouvoir et instauré le chaos, c'est la race gauloise qui a légitimité à gouverner par elle-même. Face à cette analyse polémiste, les historiens français vont privilégier une logique de concorde nationale qui insiste sur le mélange précoce des populations, tel que le décrivent François Guizot ou Frédéric Ozanam. Le succès du mot »race/Rasse« et le développement de l'anthropologie à partir de la seconde moitié du XIX ${ }^{\mathrm{e}}$ siècle confrontent les historiens au dilemme suivant: »race« est-il un équivalent de »nation« ou de »peuple«? Les observations physiques forment-elles un critère valide pour définir l'ethnicité?

Jules Michelet, lecteur attentif et admiratif de Grimm et de Herder, résume par ces termes la posture française: »La France est une nation, l'Allemagne est une race«. La population de la première est caractérisée par le mélange, la fusion, l'intégration linguistique et culturelle des migrations successives. Son unité est garantie par la forme étatique, et par une sorte de logique géographique, qui, toujours selon Michelet, dote l'espace français d'une cohérence propre. Le peuple allemand, au contraire, ne bénéficie pas d'une réalité institutionnelle, mais jouit d'une unité culturelle et linguistique interne. Les historiens allemands contemporains (Jacob Grimm) privilégient cette logique de la Völkerpsychologie: l'histoire du peuple allemand peut se reconstruire 
grâce à l'accumulation scientifique de multiples indices, linguistiques, juridiques, culturels, ethnologiques et archéologiques.

La pratique historienne du $\mathrm{XIX}^{\mathrm{e}}$ et du premier $\mathrm{XX}^{\mathrm{e}}$ siècle présente une définition du terme de »race« qui s'apparente à »famille«; à côté de celui de »Stamm« ou »tribu«, le mot correspondrait sémantiquement à la notion de »gens« et désignerait un groupe uni politiquement issu d'une même souche ethnique. Cette approche s'inscrit en filiation avec la tradition ethnographique ancienne qui, de l'Antiquité classique à la Bible, d'Isidore de Séville aux modernes, vise à décrire et à classer les peuples. L'identification de critères discriminants, qu'ils soient culturels, physiques, caractériels ou géographiques, semble permettre un affinement scientifique de cette typologie.

\section{LA FRANCE DEVANT LES RACES}

L'antiquisant Camille Jullian, élève de Fustel de Coulanges, s'est attaché à l'étude de la Gaule celtique et romaine. Son analyse historique apparaît caractéristique de l'utilisation politique de l'histoire pendant la Première Guerre mondiale et dans les années 1920. Durant cette période, ses cours du Collège de France présentent une approche continualiste de la nation française. Pourtant son nationalisme se démarque d'une approche raciste, ainsi qu'il le précise:

Ce mot de `race prédestinéeく, si volontiers prononcé outre-Rhin, nous ramène à un problème qui a souvent fixé notre attention, le problème de la race. Vous vous rappelez comment nous l'avons constamment résolu. Aussi loin que nous sommes remontés dans les temps indo-européens, nous n'avons jamais rencontré de race, c'est-à-dire d'espèce humaine physiquement, moralement, éternellement distincte; mais nous avons toujours rencontré des nations, c'est-à-dire des sociétés humaines, d'une durée plus ou moins longue, d'une étendue plus ou moins vaste, de langues et d'habitudes différentes. En d'autres termes, nous n'avons point constaté dans la vie collective des hommes des lois physiologiques, mais des institutions déterminées par le sol et par l'histoire'.

Il poursuit son propos par une dénonciation des thèses de Gustaf Kossinna (»Die deutsche Vorgeschichte«, 1912), et retourne avec ironie l'idée de »race parfaite entre toutes, la race prééminente«: selon cette logique d'immanence et de non-miscibilité, la »race allemande, distincte de celles qui se sont mêlées pour former l'Angleterre, la Belgique ou la France« serait au contraire »la race maudite, condamnée à la brutalité éternelle, issue des sombres époques de l'humanité moustérienne ou néolithique « ${ }^{2}$. Sa conscience de la dangerosité de ces raisonnements et sa volonté de s'y opposer le conduisent d'ailleurs à convoquer l'exemple de l'Afrique et à dénoncer le risque génocidaire:

Il [le mot de race] éveille une idée de conformation physique à laquelle nul n'échappe en naissant, d'habitudes matérielles que le corps nous contraint de subir, d'une inéluctable fatalité qui

1 Camille Jullian, Au seuil de notre histoire: leçons faites au Collège de France, vol. II, Paris 1919, p. 16-17.

2 Ibid., p. 18. 
pèse sur les individus et les sociétés. Il justifie les haines, les condamnations, les anéantissements même. Si vous dites que les Noirs d'Afrique sont une race inférieure, éternellement inférieure [...], vous vous résignez à sa disparition comme à une règle inévitable. Mais si vous dites, ce que je crois être la vérité, que les tribus du Soudan, par exemple, représentent la décadence actuelle de nations qui furent puissantes, civilisées, et nullement méchantes, vous émettez l'espoir que ces groupes d'hommes pourront se relever³.

Le mélange, les fusions ethniques et les phénomènes d'acculturation représentent des valeurs positives et valorisées. Ce référentiel républicain s'appuie sur la définition de la nation énoncée par Ernest Renan, quelques années auparavant: un groupe politique fondé sur une volonté commune, qui est moins soudé par un passé pluriel que par la foi en un avenir à construire ensemble. Sa conception de l'histoire et des peuples se distingue de toute notion de fatalité, elle allie au contraire libre arbitre et lois naturelles, en premier lieu celles de la géographie et du caractère. Ces deux éléments justifient un patriotisme exacerbé qui s'exprime de manière décomplexée dans un discours antigermanique. Jullian rejette l'utilisation raciste du terme de »race « - qu'il emploie par ailleurs - et remplace la notion par celle de »tempérament«, ou encore d'»âme«, de »souffle«, qui rappelle le latin anima ${ }^{4}$.

Le discours de Ferdinand Lot présente une ambivalence semblable à celle qui est observée dans celui de Jullian: l'emploi du mot »race« cohabite avec l'opposition contre toute idée de permanence et de non-miscibilité ou de caractères physiques déterminés. Rendant compte de la nouvelle édition de l'ouvrage d'Henri d'Arbois de Jubainville, »Les premiers habitants de l'Europe d'après les écrivains de l'Antiquité et les travaux des linguistes « (1894), le médiéviste s'oppose à la thèse développée selon laquelle la race française ne serait ni gauloise ni germanique, mais de souche bien plus ancienne ${ }^{5}$. Jubainville reprend en effet l'idée ancienne, défendue par certains modernes, d'une parenté celto-germanique: les Gallo-Francs formeraient en fait un seul peuple, arrivé sur le sol dit gaulois en deux vagues successives, la première préromaine - celle des Gaulois -, la seconde à partir du $\mathrm{III}^{\mathrm{e}}$ siècle - celle des Germains. François Hotman argumentait ce postulat par des éléments philologiques et politiques, il s'agissait de justifier la légitimité territoriale de la dynastie royale et l'unité de la nation. Jubainville fonde quant à lui son hypothèse sur l'anthropologie. Les Gaulois auraient été »grands et blonds ou roux «, tout comme les Germains, alors que »la majorité des Français est brune et de taille moyenne«. L'admiration que lui porte Ferdinand Lot n'empêche pas une critique ferme: ce dernier souligne le »préjugé« dans cette représentation physique. Il dénonce le postulat d'un maintien d'un »type physique aussi distinct au milieu de la population vaincue«: »Ce serait un cas unique dans l'histoire«, souligne-t-il. Il condamne enfin »l'idée fausse« selon laquelle Celtes ou Germains forment une race unique:

3 Ibid., vol. I, p. 185.

4 Ibid., vol. I, p. 186: »Il restera toujours, pour déterminer la marche d'une nation à travers le temps, il restera la nature du sol qu'elle habite et la nature de son tempérament«.

5 Ferdinand Lot, Bibliothèque de l'École des chartes (BEC) 55 (1894), p. 148-155. 
Actuellement on sait qu'aucune nation de l'Europe n'a d'unité de race, mais nous aimons à nous imaginer qu'il n'en était pas ainsi dans l'Antiquité. C'est là une erreur de perspective. Quand les Celtes apparaissent dans l'histoire, vers le $\mathrm{VI}^{\mathrm{e}}$ siècle avant notre ère, l'humanité était déjà bien vieille, et les tribus celtiques, pour avoir l'unité de langue et sans doute de mœurs, n'en étaient pas moins la fusion de bien des races préexistantes à jamais indiscernables ${ }^{6}$.

En 1913, Lot plaide de nouveau pour une »science de l'histoire ethnique« qui n'aurait »rien à voir avec le gobineauisme « ${ }^{7}$. Le royaume mérovingien se caractérise par la $»$ confusion des races $\ll^{8}$, c'est-à-dire le mélange sans regard pour cette distinction. Au $\mathrm{VII}^{\mathrm{e}}$ siècle déjà le terme de francus désigne tout homme libre, sans référence aucune à son origine. Si l'assimilation ne s'effectue pas de manière homogène selon les territoires, ses formes diverses annoncent les particularismes régionaux, mais ne remettent pas en question la fusion totale qui s'est effectuée entre »indigènes« et »envahisseurs«. L'acculturation est favorisée par l'union confessionnelle avec la conversion de Clovis, le choix du latin comme langue administrative, l'absence d'usurpation de terre. La thèse d'un apport démographique restreint, tant numériquement que géographiquement (limité à la partie septentrionale de la Gaule) conforte l'idée d'une intégration rapide des populations. La préservation de caractères spécifiques n'aurait été possible, selon Lot, que si les Germains de Clovis avaient eu une volonté délibérée de ne pas se mélanger aux Gallo-Romains. Or, leur désir consistait précisément à intégrer le modèle impérial, même si cet effort initiat en fait une décadence de celui-ci.

L'égalité politique des habitants du Regnum Francorum a frappé tous les historiens. Tout habitant de la Gaule, qu'il soit Franc, Romain, Bourguignon, libre ou affranchi, riche ou pauvre, peut entrer au service de l'État et faire une carrière. Tous sont égaux politiquement, parce qu'ils sont égaux dans la servitude. Le Mérovingien n'a point de système, point de préjugé de race, de langue, de condition sociale9.

Le maintien de l'emploi de »race« dans le sens d'origine politique (l'expression »race des Francs « revient fréquemment) relève donc de l'usage et ne prête pas à confusion. En 1947, dans son ouvrage »La Gaule. Les fondements ethniques, sociaux et politiques de la nation française«, le mot apparaît régulièrement. La fusion de races diverses, indigènes et exogènes, forme le creuset français, et inscrit par le sol une continuité de la nation. Lot se démarque des dérives récentes, il écrit: $» I l$ ne faut jamais oublier - et anthropologues et préhistoriens l'oublient constamment - qu'un squelette ne nous fait jamais la grâce de révéler sa nationalité «10. La bibliographie proposée à la fin de son ouvrage reflète son incapacité à renvoyer à des travaux scientifiquement irréprochables, et s'il cite Broca, Collignon, Topinard et Montandon, il en souligne les lacunes;

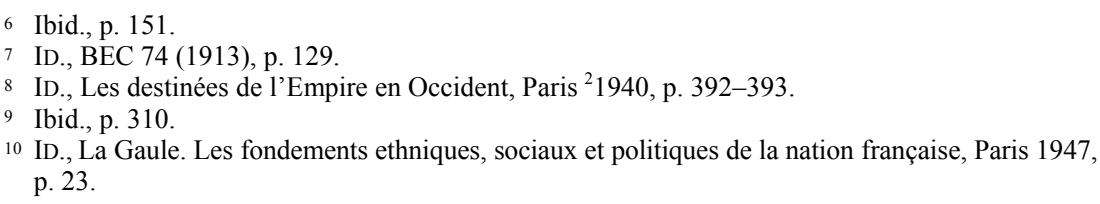


de la même manière, sa référence au »Germanische Volkserben in Wallonien und Nord-Frankreich« de Franz Petri (Bonn 1937) se double d'une prévention. Le dessein de l'ouvrage semble être, sans polémique et sans xénophobie, de vulgariser la thèse d'une continuité gallo-française et de l'existence d'un type français, tant physique que moral, qui se distingue du type germano-allemand, et ceci sur l'ensemble du territoire national ${ }^{11}$. L'auteur rejoint ainsi finalement l'idée d'un »tempérament « français développée par Camille Jullian.

Dans ses écrits sur les invasions, l'historien Marc Bloch appréhende les mondes germanique et romain comme deux entités distinctes (même si elles ne sont pas fermées), c'est-à-dire comme deux modèles culturels ou "stades de civilisation«. L'approche ethnique est nécessaire pour appréhender les structures et la notion de »choc civilisationnel «12. Les deux peuples sont définis culturellement, et non biologiquement: »Leur antagonisme fut moins, en somme, le heurt de deux traditions ethniques différentes que celui de deux types de sociétés parvenues, chacun, à des stades inégaux d'évolution. [...] Des institutions plus archaïques se trouvèrent face à face avec des institutions d'un modèle plus évolué; et les premières souvent triomphèrent des secondes $\aleph^{13}$. Dans »Les caractères originaux de l'histoire rurale française «, il qualifie la thèse d'un lien entre occupation du sol et race d'»incroyablement simpliste « ${ }^{14}$. Il s'en démarque ainsi:

Au temps où la race semblait devoir donner la clef du passé, on songea, tout naturellement, à demander au Volksgeist le mot de cette énigme, comme de tant d'autres. Tel fut notamment, hors de France, l'objet de la grande tentative de Meitzen, précieuse comme initiatrice, mais qu'on doit tenir aujourd'hui pour définitivement ruinée. Aussi bien, entre autres torts, avait-elle celui de ne tenir compte que des peuples historiquement attestés [...]. Mais ne parlons ni de race ni de peuple: rien de plus obscur que la notion d'unité ethnographique. Mieux vaut dire: types de civilisation ${ }^{15}$.

À ces »deux types de civilisations« se rattachent »deux structures économiques «16: l'opposition n'est pas de nature mais de degré. La crise monétaire, politique et sociale

11 Ibid., p. 25: »le fait que le Gaulois historique est l'ancêtre du Français et physiquement et moralement dans l'immense majorité des cas «.

12 Marc BlOCH, Les invasions barbares, dans: Revue de Synthèse historique 60 (1945), p. 105 : »Quoi qu'en ait pensé le bon abbé Dubos, quoi que Fustel lui-même ait parfois paru indiquer, il est décidément impossible de concevoir l'invasion sous les idylliques couleurs d'une suite d'alliances entre peuples conquérants et peuples conquis«.

13 ID., La société du haut Moyen Âge, dans: Journal des savants (1926), p. 71, où il tente de remettre »l'accent sur les haines de peuple à peuple« qui, de surestimées par l'historiographie nationaliste, lui paraissent maintenant sous-estimées: elles constituent pourtant, selon l'historien, un fait historique, très présent dans les sources, et non négligeable.

14 Ibid., p. 66: sur les »partis pris ethnographiques de Meitzen«, c'est-à-dire »une thèse invraisemblablement simpliste, dont un des postulats essentiels est d'admettre que les Germains ont possédé, comme un signe de race, un mode d'établissement spécifique«.

15 ID., Les caractères originaux de l'histoire rurale française, Paris ${ }^{2} 1952$, p. 102.

16 ID., Une mise au point: les invasions, Annales HS, 1945, vol. 1, p. 33-46 et vol. II, p. 13-28; ID., Mélanges historiques, vol. I, Paris ${ }^{2} 1963$, p. 110-141. 
du III $^{\mathrm{e}}$ siècle forme la raison structurelle de la déstabilisation de l'Empire et du succès barbare ${ }^{17}$; parallèlement, le ralliement des populations gallo-romaines au pouvoir franc s'explique par des facteurs économiques et politiques. Il rejette la thèse d'une typologie raciale d'occupation du sol, mais admet les lectures soit régionales, soit historiques, pour lesquelles la toponymie représente un fossile de cet apport démographique linguistiquement distinct. L'ensemble des territoires gaulois et germains de la basse Antiquité et du haut Moyen Âge se caractérise par une occupation très lâche du sol et par une structure agraire »trouée« de zones dépourvues de peuplement. Les déplacements de populations s'appréhendent en deux temps: »La mise en marche de peuplades entières avait d'ailleurs été précédée, en Germanie, par une lente migration qu'atteste l'inflation à travers le monde romain de tant d'éléments germaniques, à tous les degrés de la hiérarchie sociale«. À la suite de ces migrations diffuses succède un déplacement de population très important (en regard de la pression démographique de l'époque, s'entend ${ }^{18}$ ) au moment même des conquêtes (deuxième moitié du $\mathrm{V}^{\mathrm{e}}$ siècle), selon un mouvement qui ne concernerait donc pas de simples armées, mais des populations complètes. Les Francs Saliens s'installent »dans la plaine basse au sud des bouches de l'Escaut, Flandres, Brabant occidental et septentrional«, au sein de vides »qu'ils avaient eux-mêmes créés« par leurs incursions dévastatrices précédentes, et repeuplent ainsi presque totalement le pays ${ }^{19}$. L'adhésion de Marc Bloch à la thèse d'une migration démographiquement significative, en opposition donc avec les analyses de Camille Jullian et de Ferdinand Lot, ne l'incitent pas pour autant à adopter l'approche de la Rassenkunde. Son refus de la logique ethnique ne s'appuie pas sur des arguments politiques ou sur la simple historicisation, mais sur l'explication socioéconomique. La revue les »Annales«, qu'il a fondé et codirige, s'illustre par ailleurs tout au long des années 1930 par une conscience vive des dérives de l'archéo-anthropologie allemande contemporaine ${ }^{20}$.

17 Ibid., p. 111

18 Ibid., p. 130: „Vinrent les invasions germaniques. À l'échelle d'aujourd'hui, les déplacements d'hommes qu'elles provoquent paraîtraient sans doute assez médiocres«.

19 Ibid., p. 131: »Les Francs Saliens paraissaient bien n'avoir rencontré que des établissements de tout temps très clairsemés; leur arrivée peupla véritablement le pays et prépara ainsi sa future grandeur économique, que devait favoriser sa place au centre même des communications de la nouvelle Europe«.

$20 »$ Pendant ce temps-là, un pauvre diable d'instituteur romagnol, phobique, plein d'idées de sorcellerie, de superstitions, d'absurdités apprises et héritées, et un peintre en bâtiment inculte et ignare des Alpes autrichiennes, avec dans la tête ce que nous pouvons y supposer également d'idées préhistoriques naïvement transposées, vouent le monde à l'anéantissement«, résume Lucien Febvre dans une lettre à Marc Bloch (mars 1939) dans: Bertrand MÜLlER (éd.), Correspondance III. Les »Annales« en crises (1938-1943), Paris 2003, p. 58. 


\section{LA TENTATION DE LA RACIOLOGIE EN ALLEMAGNE »UNE UNITÉ RACIALE HISTORIQUEMENT FONDÉE«}

Les dernières années du XIX ${ }^{\mathrm{e}}$ siècle avaient vu une focalisation renforcée de l'intérêt des historiens allemands sur leur Vorgeschichte, de la préhistoire à Charlemagne. Après le moment romantique de l'exaltation de l'âme allemande sous la plume de Grimm, pour fonder la nation allemande en un peuple historique doté d'une langue, d'une culture, d'un droit et de caractères particuliers et communs, l'effort de la jeune Verfassungsgeschichte, sous l'égide de Georg Waitz, avait consisté en une légitimation de la culture germanique ancienne et médiévale: ceux que l'historiographie française se plaisait à nommer Barbares formaient selon ce nouveau paradigme une société organisée, dont il était désormais possible de décrire les structures et les règles. Les synthèses proposées par Felix Dahn et par Walther Schultze au tournant du siècle approfondissaient ce constat en insistant, pour la première, sur la continuité de la culture allemande, et, pour la seconde, sur son évolution historique, dans laquelle la fusion avec la romanité et la conversion au christianisme constituaient un apport essentiel. L'un comme l'autre présentaient un tableau du Germain aux caractères physiques et moraux bien déterminés, que le nationalisme prussien, renforcé par la récente refondation du Reich en 1870, contribuait à promouvoir. Cette invention de la figure de l'Allemand éternel se trouve déjà dans le premier volume de la »Geschichte des deutschen Volkes « d'Eduard Duller (1840), qui en fait la description suivante: „Gross, stark und schön waren die Deutschen in alter Zeit. Weiss und rein war die Farbe ihrer Haut, in üppiger Fülle floss das goldgelbe Haar, der Mähne des Löwen ähnlich, bei Männern und Frauen hernieder, und aus den grossen blauen Augen blickten Muth und edler Freiheitsstolz « ${ }^{21}$.

Face au succès de la race active germanique ou aryenne qui se développe chez les pangermanistes, Franz Steinbach s'interroge sur la notion de Stamm et semble rejeter celle de Rasse. Son ouvrage "Studien zur Westdeutschen Stammes- und Volksgeschichte« (1926) propose d'intégrer les trois indices linguistique (Dialekte), toponymique (Ortsnamen) et architectural (Bauernhausformen) susceptibles d'identifier culturellement les peuplades (Stämme) et d'évaluer à partir de ceux-ci la question de la frontière occidentale entre peuples romans et peuples germaniques. Cependant, le projet de son étude ne consiste pas seulement à proposer deux morphologies culturelles (Kulturmorphologie) opposées. Il vise aussi à résoudre la question de l'historicité de cette opposition, et de l'influence des migrations barbares sur celle-ci. L'historien souhaite contrecarrer les deux théories suivantes. Pour la première (défendue par Auguste Longnon), la France ressemble à »une œuvre d'art peinte par la main d'un

21 »Les Allemands du temps passé étaient grands, forts et beaux. La couleur de leur peau était d'un blanc pur et leur chevelure dorée se répandait en ondulant, telle la crinière du lion, tant chez les hommes que chez les femmes, et de leur grands yeux bleus perçait un regard noble empli de courage et de fière liberté«, Eduard DulLER, Geschichte des deutschen Volkes I, Berlin 1840 , p. 6. 
maître « ${ }^{22}$, la monarchie franque puis française, qui a uni les peuples tels des matériaux naturels dans un espace destiné à former une unité; pour la seconde (celle de Johannes Haller), le peuple allemand est le produit de l'État ${ }^{23}$. Il ne procède pas d'une unité naturelle mais d'une construction historique, et sa naissance n'est pas antérieure au $\mathrm{X}^{\mathrm{e}}$ siècle. À la place du politique et de l'institutionnel comme élément déterminant dans la formation nationale, Franz Steinbach souhaite placer ce qu'il nomme le particularisme de chaque peuple. Mais son approche se distingue, et de l'ancienne vision romantique, qui lie de manière intrinsèque un peuple à sa langue ${ }^{24}$, et de la nouvelle analyse de la Rassenkunde, qui fonde celui-ci sur une détermination biologique préservée à travers l'histoire. La fusion culturelle est possible sans fusion de race, car celle-ci correspond à un processus beaucoup plus long: les recherches anthropologiques ont montré que le contact ethnographique précède le mélange anthropologique, c'est-à-dire qu'une communauté de langue et de culture peut se former sans fusion des $\operatorname{races}^{25}$. Cet apport permet d'affirmer que la langue et l'ethnos ne sont pas liés; une langue seule ne forme pas une nationalité, même si elle reste un composant essentiel de l'unité culturelle ${ }^{26}$. Tout en reprenant cette thèse, Franz Steinbach se distingue de la logique raciale en refusant de donner à l'habitus ethnique (racial) une place prépondérante par rapport à l'habitus culturel. L'application du concept de race reviendrait à reconnaître dans chaque pays européen une extraordinaire mosaïque ethnique (Rassenmosaik), »au moins six [ethnies] différentes pour l'Allemagne dès le premier coup d'œil«, et la logique du principe défendu par Gustaf Kossinna entraînerait la détermination de délimitations géographiques fantaisistes, fondées sur ce qu'il nomme la race ${ }^{27}$. C'est l'emploi même du mot Rasse qui rend, pour le lecteur contemporain, la position de Franz Steinbach équivoque, alors même qu'il définit le terme, en lien avec celui de

22 Auguste Longnon, »Origines et formation de la nationalité française. Eléments ethniques-Unité territoriale«, leçon proférée au Collège de France en 1889, publiée dans La formation de l'unité française, 1922; cité par Franz STEINBACH, Studien zur westdeutschen Stammes- und Volksgeschichte, Iéna 1926, p. 1: »Gemälde von Meisterhand«.

23 Johannes HALLER, Die Epochen der deutschen Geschichte, 1923, cité par STEINBACH, Studien (voir n. 22), p. 1: »Nach ihm gibt es vor dem 10. Jahrhundert keine deutsche Geschichte und >das deutsche Volk ist keine natürliche, sondern eine geschichtlich gewordene Einheit««.

24 STEINBACH, Studien (voir n. 22), p. 16: »Zwar setzt man meist nicht mehr im Sinne der romantischen Schule von Jakob Grimm und wie noch Arnold Sprache und Rasse als untrennbar voraus«.

25 Ibid., p. 14: il reprend là essentiellement l'analyse de RATZEL, Anthropogeographie I, Stuttgart 1882, p. 394, mais se réfère aussi à Paul VIDAL DE LA BLACHE, Principes de géographie humaine, Paris 1922, notamment p. 277, et, assez logiquement, à Camille JULLIAN, De la Gaule à la France, Paris 1922, chap. II.

26 SteINBACH, Studien (voir n. 22), p. 16: »eine Sprache allein begründet kein Volkstum; aber sie [ist] doch wenigstens eine der wichtigsten Komponenten der kulturellen Einheit«.

27 Ibid., p. 17: L'auteur évoque là l'ouvrage de Gustaf KossinNA, Herkunft der Germanen, Wurtzbourg 1911. 
Stämme, comme une »unité raciale historiquement formée« plutôt que comme une stricte »origine commune $\ll^{28}$.

La construction franque devient une réalisation politique qui ne procède d'aucune réalité ethnique: »les Francs ne sont pas nommés en tant que tribu germanique«, l'origine et la signification de leur nom même est extrêmement confuse ${ }^{29}$. Il apparaît inutile de tenter d'identifier historiquement leur formation préhistorique: les Ripuaires, selon l'auteur, ne sont pas une peuplade aux caractères spécifiques, un Stamm (»keine geschlossen eingerückte Völkerschaft, kein Stamm«), mais une création politique nouvelle du $\mathrm{V}^{\mathrm{e}}$ siècle. Les Alamans n'ont pas davantage d'origine commune ${ }^{30}$. Le rôle de Clovis puis de ses fils en tant qu'unificateurs des peuples (Stämme) germaniques constitue un fait politique incontestable et relève bien d'une »véritable construction étatique dynastique «31. Les tribus allemandes (deutsche Stämme) sont des constructions socio-historiques, des unités culturelles qui procèdent de l'influence réciproque du cadre géographique et de l'histoire: la conscience tribale ou régionale (Stammesbewußtsein) dépend strictement d'un espace donné32. Ce constat ne remet pas en cause l'ancienneté de ces »unités historiquement construites« (»geschichtlich gewordene Einheiten «), mais au contraire, par la définition non strictement politique mais également sociale et culturelle, leur offre une nouvelle légitimité historique. Il intégre la notion de temps et de mutation culturelle, et dépasse ainsi à la fois la lecture linéaire raciale, invalide scientifiquement, et la lecture historico-politique, qui ne prend en compte que les formes étatiques. »Aussi longtemps que le peuple allemand vivra, les tribus continueront dans son sein leur croissance et leurs mutations $\ll^{33}$. Concernant la fin du Bas-Empire et le début du Moyen Âge donc, »l'élément primordial de la construction tribale après la confusion de la période migratoire n'a pas été la différenciation raciale et ethnique des populations germaniques en des groupes fermés, ni la naissance de spécificités qui s'affirmeront au cours des temps, mais l'élaboration de particularismes politiques et territoriaux «, qui participent à la réalisation mérovingienne ${ }^{34}$. Le temps des migrations ne signifie pas une rupture totale au sein de l'évolution culturelle européenne, et les Germains ne pénètrent pas comme des peuplades fermées (»als geschlossene Stämme«) dotées de caractères définitifs dans l'espace occidental. La frontière linguistique ne constitue pas une relique de la période des migrations, mais

28 Ibid., p. 15: »Es ist heute bei den Historikern zweifellos noch sehr üblich, gerade in den blutsmäßigen Anlagen und Eigenschaften, also in der Rasse das Wesen des Volkstums zu sehen. Das unglückselige Wort `Stämme` wirkt in derselben Richtung, obwohl man weniger an gemeinsame Abstammung als an geschichtlich gewordene Rasseneinheit denkt «.

29 Ibid., p. 116.

30 Ibid., p. 117.

31 Ibid., p. 115.

32 Ibid., p. 123: »Die Stämme sind geschichtlich-soziale Bildungen, kulturelle Einheiten, entstanden durch Wechselwirkung von Raum und Geschichte im weitesten Sinne des Wortes. Darum hat sich ein geschlossenes Stammesbewußtsein nur in bestimmten Landesteilen entwickelt «.

33 Ibid., p. 124.

34 Ibid. 
une démarcation claire qui sépare deux grands espaces culturels distincts ${ }^{35}$ : leurs différences apparaissent antérieures à la Völkerwanderung, même si celle-ci les renforce et que le temps va progressivement les conforter. Ils forment les deux communautés culturelles appelées peuple allemand et peuple français, bien avant le $\mathrm{X}^{\mathrm{e}}$ siècle. Franz Steinbach défend ainsi le caractère allemand de la région rhénane sur la base d'une unité du peuple allemand fondée moins sur la race que sur la culture et la langue, sachant que celle-ci n'est pas une compétence intrinsèque mais un produit de la culture, notamment de l'éducation. Trois éléments structurent cette analyse: premièrement l'idée du Stammesbewußtsein (conscience tribale) et de ses manifestations culturelles; deuxièmement la frontière linguistique, produit de l'évolution culturelle; troisièmement la particularité, culturelle donc, du caractère allemand (Deutschtum) antérieure à la naissance institutionnelle de l'État allemand ${ }^{36}$, et en opposition fondamentale avec l'identité française.

De récents travaux consacrés à la communauté historienne allemande pendant le nazisme ont rappelé les difficultés administratives et scientifiques auxquelles furent confrontés les historiens: plusieurs sont contraints à la démission ou à l'immigration, et les jeunes habilités hostiles au régime doivent privilégier des carrières annexes, et prennent notamment des postes d'archivistes qui leur évitent l'adhésion au parti nazi, obligatoire pour obtenir un poste à l'Université. Ludwig Schmidt, directeur des archives de Dresde, bénéficie à ce titre d'une situation privilégiée: dès la fin des années 1920, il a acquis une reconnaissance internationale en matière d'analyse de la Völkerwanderung et propose pourtant une approche nuancée par rapport au diktat de la Rassenkunde et de l'école archéo-anthropologique de Gustaf Kossinna. Sa »Geschichte der germanischen Frühzeit«, publiée en 1925 et rééditée en 1934, énonce ainsi les points suivants: les Germains des premiers siècles ne forment pas un peuple, mais seulement une branche ethnique (Stamm) $)^{37}$. Ils ne correspondent pas aux Allemands (Deutsche), puisque le mot même de deutsch n'apparaît qu'au IX ${ }^{\mathrm{e}}$ siècle. La localisation de leur territoire d'origine (Urheimat) pose problème: l'historien relate les hypothèses successives qui privilégient soit l'Asie centrale, soit l'Europe septentrionale, sans pour autant privilégier l'une ou l'autre, ce qui représente en soi une remise en cause de la thèse nordique.

Suchte man dieselbe [Heimat] früher allgemein in Zentralasien, so hat man sich neuerdings auf Grund sprachwissenschaftlicher, archäologischer und anthropologischer Kriterien für Europa insbesondere Nordeuropa (Südscandinavien, Dänemark und Norddeutschland) entschieden; aber es mehren sich in neuerer Zeit die Stimmen, die wieder für asiastische (osteuropäische) Sitze

35 Ibid., p. 179: »dass die Sprachgrenze nicht ein zufälliges Relikt der Völkerwanderung ist, sondern die klare Grenzlinie zweier großer Kulturzentren darstellt, die nach der Verwirrung der Völkerwanderungszeit sich immer schärfer gegeneinander abgesetzt haben«.

36 Ibid., p. 180: »Dieser Aussonderungsprozeß des Deutschtums liegt vor der Bildung des deutschen Staates«. Le moment carolingien est assimilé à une étape au cours de ce processus.

37 Ludwig SCHMIDT, Geschichte der germanischen Frühzeit. Der Entwicklungsgang der germanischen Nation bis zur Begründung der fränkischen Universalmonarchie durch Chlodowech, Cologne ${ }^{2} 1934$, p. 3 . 
eintreten und auf die Unsicherheit der für die nordeuropäische Theorie angeführten Gründe (Vorkommen botanischer Namen, Kenntnis des Meeres u.a.) hinweisen ${ }^{38}$.

La description du peuple germanique, très détaillée, reprend en partie les canons essentiels de la tradition germaniste, tant sur le plan physique que moral (la taille, les indices corporels, l'amour de la liberté, le courage, la discipline militaire). »Nombre de ces indices se sont maintenus jusqu'à aujourd'hui, notamment dans le Nord « ${ }^{39}$, indique Schmidt, qui précise qu'»en Allemagne méridionale le type brun maintenant domine, $\mathrm{du}$ fait de l'influence des modifications des conditions de vie et du climat, mais aussi du mélange avec les Celtes«. Plus spécifiquement, il annonce ne pas préférer se prononcer sur le degré d'indépendance des relations des Germains vis-à-vis des mélanges raciaux ${ }^{40}$.

La perspective choisie par Ludwig Schmidt reste définitivement historicisante: les Germains ne sont pas des sauvages, mais, au début des migrations, leur niveau culturel (Kulturstufe) apparaît effectivement en deçà de leurs contemporains romains. Ce décalage provient de raisons climatiques, et par contre leur grande capacité culturelle (Kulturfähigkeit) s'exprime durant la Völkerwanderung, au cours de laquelle ils rencontrent la civilisation romaine et s'intègrent très rapidement à elle par un effet de fusion ${ }^{41}$. L'insistance sur cette notion de mélange témoigne tant de l'opposition de l'auteur aux théories des raciologues que de l'influence des thèses contemporaines d'Alfons Dopsch, et, au-delà, de l'apport de la jeune sociologie.

Euvre d'un jeune historien rhénan déjà reconnu, qui continuera sa carrière universitaire après guerre, le Volkserbe de Franz Petri offre au contraire une lecture bien plus paradoxale, et ce à deux niveaux: méthodologiquement, il revendique la convocation de la raciologie dans l'analyse historique du peuplement germanique de l'espace franc et énonce l'apport de ses propres travaux d'historien à cette même raciologie ${ }^{42}$; scientifiquement, il développe une thèse radicale et nouvelle de la question, sur laquelle il se rétractera ensuite (»Die fränkische Landnahme und die Enstehung der germanischromanischen Sprachgrenze in der interdisziplinären Diskussion«, 1977). L'impact démographique et géographique des migrations dites germaniques du Bas-Empire aurait été largement sous-estimé. La présence multiple de traces (archéologiques, linguistiques, toponymiques, culturelles, raciales) bien en deçà de la frontière linguistique permet au contraire d'affirmer une implantation large et profonde dans une large moitié septentrionale. Son souvenir s'est trouvé comme effacé par le processus de reromanisation des territoires qui lui a succédé. Une cartographie nouvelle de l'espace »germanique« altimédiéval se dessine alors, dont la taille apparaît bien supérieure à celle

38 Ibid., p. 4.

39 Ibid., p. 13.

40 Ibid., p. 12: »Inwieweit die somatischen Verhältnisse der Germanen von der Rassenmischung abhängig sind, vermögen wir nicht zu sagen«.

${ }^{41}$ Ibid., p. 161.

42 Franz PETRI, Germanisches Volkserbe in Wallonien und Nordfrankreich, Bonn 1937, p. 853: »Das instruktivste unter ihnen ist die Rassenforschung mit ihren Ergebnissen über die Verbreitung der großen europäischen Rassen«. 
définie dans les analyses traditionnelles. La prise du pouvoir mérovingienne ne constitue donc pas un miracle historique, comme les historiens français la décrivent, mais le succès d'un peuple fortement implanté dans un large espace franc. Le substrat ethnique forme une réalité endémique face aux mutations politiques de romanisation, germanisation, re-romanisation successives. La forme même de cette installation primitive, majoritairement paysanne, constitue un argument supplémentaire en faveur d'une implantation souche. L'espace entre Seine et Loire se caractérise donc par une installation germanique importante (»eine wirkliche germanische Volkssiedlung«), contrairement aux postulats de l'historiographie antérieure et contemporaine qui s'accordait à limiter au nord de la Seine une installation germanique plus ou moins significative ${ }^{43}$. Après avoir rappelé la définition physique des divers types raciaux et les critères utilisés (»Körpergröße, Haut- und Haarfarbe und Schädelindex« notamment), Petri conclut:

Die Zusammenfassung der verschiedenen Rassenbilder [...] liefert ein höchst merkwürdiges Ergebnis: Genau diejenigen Gebiete, die sich uns auf Grund der Kombinierung von Namenforschung und frühmittelalterlicher Archäologie als Hauptverbreitungsgebiete der fränkischen Landnahme herauskristallisiert haben, erscheinen auf der Rassenkarte als südwestlichstes Verbreitungsgebiet des nordischen Typus. Die Übereinstimmung ist geradezu frappierend. Von dem Reliktgebiet in den Ardennen abgesehen, hebet sich das ganze Frankenreich Chlodwigs auch rassisch als eine Einheit heraus. Untere Seine und das Loireknie bei Orleans erscheinen als nordische Rassengrenze.

Et cette observation explique la vitesse du succès politique franc, ainsi que »la dissolution raciale rapide des Celtes $«{ }^{44}$. L'utilisation contemporaine de ces recherches dans le cadre de la Volksgeschichte telle que la prône l'historien Adolf Helbok (»Grundlagen der Volksgeschichte«, 1937), fondée sur la théorie du Blut und Boden et sur celle des peupliers, mais également sa captation par les pouvoirs politiques comme justification de la guerre puis de l'annexion de territoires dits ethniquement germaniques, rend ce discours particulièrement sujet à doutes quant au dessein initial de l'historien.

\section{UNE CONCEPTION HISTORIQUE DE LA RACE}

L'étude comparative des discours des historiens français et allemands montre globalement la défense d'une conception spécifiquement historique de la race. Le terme, dans le sens de famille ethnique, est toujours un produit politique et culturel en perpétuelle mutation. La tradition micheletienne (»et ta race est $89 «^{45}$ ), reprise par Jullian, associe le mot à celui de liberté. Le point de vue allemand reste marqué par la référence à un type germanique originel et prédominant, mais dont les historiens soulignent le caractère construit (il s'agit d'une représentation autant que d'une réalité) et historiquement

43 Ibid., p. 854.

44 Ibid., p. 856.

45 Jules Michelet, Bible de l'humanité, Paris 1864, conclusion; voir l'analyse de ce texte par Claude RÉTAT, »Jules Michelet, l'idéologie du vivant«, dans: Romantisme 130 (2005), p. 9-22. 
dépassé. La notion de mélange, rappelée par tous, s'oppose à la conception raciste, fondamentalement anachronique et contraire à tout effort d'historicisation. Pourtant, l'impact de la raciologie s'observe dans la définition de plus en plus généralisée de faciès ou de types ethniques chez les historiens, et certains apports de l'anthropologie et de la raciologie sont amplement cités. Une partie de l'historiographie allemande présente une contamination progressive des idées des raciologues, en premier lieu celle de la non-miscibilité. Parallèlement, les travaux d'historiens servent de réservoir à idées et à preuves pour la raciologie: l'ouvrage de Franz Petri permit l'élaboration chez Rudolf Graemer ou Adolf Helbok d'une logique historique de la domination de la race aryenne. La cartographie de la présence germanique au début du haut Moyen Âge devient celle d'une population contemporaine dite aryenne - celle du Troisième Reich à construire -, et l'espace dynamique de la Völkerwanderung se transforme, sous la plume des théoriciens nazis, en zone légitime de l'espace de domination allemande.

La résistance de l'historiographie aux thèses raciales, et éventuellement la dénonciation scientifique de celles-ci, a coexisté avec une contamination linguistique des discours (emploi du terme et utilisation de la lecture ethnique dans la majorité des écrits). Le démantèlement institutionnel de la communauté historienne en Allemagne dès 1933 a limité la capacité de celle-ci à exercer un contrôle scientifique des discours. Si les historiens postulent toujours une conception historique de la notion de race, la dérive »raciste« contemporaine rend difficile le respect d'une déontologie de la science historique et permet le réinvestissement pervers du travail des historiens par les raciologues, notamment en Allemagne. Seul le choix d'une approche socioéconomique, ainsi que l'a tentée Marc Bloch, permet de contrer les dérives d'une lecture ethnique. Ce constat oblige, après la Seconde Guerre mondiale, à une expulsion définitive du terme des discours: celui d'»ethnie«, euphémisme dépourvu de connotation, le remplace, sans qu'une véritable mise au point sémantique ne s'opère, puisque ce mot peut désigner à la fois la »race historique«, la tribu (c'est-à-dire un peuple dépourvu de réalité institutionnelle) ou encore une unité culturelle et/ou linguistique ${ }^{46}$.

Antiquisants et médiévistes français et allemands de ce début du $\mathrm{XX}^{\mathrm{e}}$ siècle semblent ainsi avoir globalement poursuivi un combat scientifique contre les préjugés sur la race et l'utilisation fallacieuse de ce concept. Ils l'ont mené alors que la perspective d'un renouvellement des savoirs par l'anthropologie et l'archéologie leur apparaissait une opportunité exceptionnelle pour comprendre et trancher les problématiques questions de la démographie antique et altimédiévale, et des mouvements de population. C'est à cet espoir désabusé que Ferdinand Lot en appelait en 1894:

C'est l'anthropologie qui devrait avoir le dernier mot. Jusqu'ici, malheureusement, les travaux ont été conduits, du moins chez nous, sans méthode critique et sous l'empire d'idées préconçues. Tout est à refaire. Du train où vont les choses en France, il est à craindre qu'on ne s'y mette pas sérieusement avant le $\mathrm{XXI}^{\mathrm{e}}$ siècle ${ }^{47}$.

46 Alice KRIEG-PlanQUe, Le mot »ethnie«: nommer autrui. Origine et fonctionnement du terme »ethnie« dans l'univers discursif français, dans: Cahiers de lexicologie 87 (2005), p. 141-161.

47 LOT, BEC (voir n. 5), p. 155. 